\title{
Avaliação das Técnicas OORT para Inspeção de Especificações de Requisitos em UML: um estudo empírico
}

\author{
Tereza G. Kirner, Erik R. da Cruz, Maria Imaculada Montebelo \\ Universidade Metodista da Piracicaba \\ Programa de Mestrado em Ciência da Computação - FCMNTI \\ Rodovia do Açúcar, Km 156 - 13400-911, Piracicaba - SP \\ \{tgkirner, milmonte\}@unimep.br, cruz@claretianas.com.br
}

\begin{abstract}
The use of the Unified Modeling Language (UML) has been expanding significantly in the companies. However, given the great amount of information generated through the diagrams, it is essential to develop programs for the evaluation of UML models, aiming to detect and eliminate defects that could be inserted in the system specification. One of the most efficient methods for the evaluation of software artifacts is the inspection. This work presents an empirical study on the evaluation of Object-Oriented Reading Techniques (OORTs) for inspecting specifications in UML, performed with the objective of evaluating the efficiency of such techniques as well as some issues related to professional practice.
\end{abstract}

Resumo. $O$ uso da Unified Modeling Language (UML) vem se expandindo significativamente no ambiente empresarial. No entanto, dada a grande quantidade de informação contida nos diagramas, é primordial que se invista na avaliação dos modelos UML, a fim de se detectar e eliminar defeitos existentes na especificação do software. Um dos métodos mais eficientes para a avaliação de artefatos de software, que visa detectar defeitos, que deverão ser posteriormente removidos, é a inspeção. Este trabalho apresenta um estudo empírico sobre a avaliação das Object-Oriented Reading Techniques (OORTs) para inspeção de especificações preparadas em UML, conduzido com o objetivo de avaliar a eficiência das técnicas e fatores relacionados à utilização das mesmas por profissionais da área.

\section{Introdução}

A UML (Unified Modeling Language) teve sua origem nos conceitos e notações da orientação a objeto e seu objetivo é criar modelos gráficos representativos de sistemas de software. Os nove diagramas oferecidos pela UML possibilitam a produção da modelagem conceitual e do projeto de alto nível do sistema [Booch, 1999]. Sua utilização vem se expandindo, não apenas no ambiente acadêmico como também nas empresas, em todo o mundo. No entanto, dada a grande quantidade de informação gerada através dos diagramas e a forte inter-relação existente entre os diferentes 


\section{Simpósio Brasileiro de Qualidade de Software}

diagramas, é fundamental que se invista na avaliação dos modelos UML, de forma a se detectar e eliminar defeitos inseridos na modelagem e projeto avaliados.

Os principais defeitos que podem ocorrer em modelos UML são inconsistências, omissões e ambigüidades, referentes a um único diagrama, a dois ou mais diagramas analisados comparativamente, ou a diagramas em relação ao Documento de Requisitos do Sistema [Travassos, 1999]. Tais defeitos, se não forem devidamente identificados e eliminados, levarão a falhas que prejudicarão a qualidade dos modelos UML, podendo resultar em sérios prejuízos à qualidade do produto desenvolvido.

Um dos métodos mais eficientes para a avaliação de artefatos de software, visando detectar defeitos que deverão ser posteriormente removidos ou corrigidos, é a inspeção. A inspeção de software foi proposta inicialmente por Fagan (1976, 1986), sendo aprimorada, ao longo do tempo, por outros autores [Gilb, 1992; Porter, 1996]. Tradicionalmente, o processo de inspeção tem, como elemento chave, sessões ou reuniões nas quais especialistas trabalham em conjunto na detecção de defeitos incorporados nos artefatos analisados. Como exemplos de artefatos de software passíveis de serem inspecionados, destacam-se: documentos de requisitos de software; especificações de requisitos expressadas em uma linguagem ou método, como análise estruturada ou orientação a objetos; código do software, em determinada linguagem de programação; etc.

Um processo de inspeção direcionado especificamente para modelos UML foi proposto por pesquisadores na Universidade de Maryland, Estados Unidos. Este processo - Object-Oriented Reading Techniques (OORTs), compõe-se de sete técnicas de leitura que devem ser empregadas conjuntamente. Em cada técnica, ou dois diagramas UML são avaliados comparativamente ou um diagrama é avaliado em relação ao Documento de Requisitos de Software [Travassos, 1999]. As técnicas OORT já foram empregadas, com sucesso, por outros autores, conforme discutido em Bunde (2002) e Conrad (2003).

Este trabalho apresenta um estudo empírico sobre a avaliação das técnicas OORT, conduzido com o objetivo de avaliar tanto a eficiência das técnicas, em termos de detecção de defeitos, quanto fatores relacionados à utilização das técnicas por analistas de sistemas de instituições brasileiras da região de Piracicaba, estado de São Paulo. O trabalho relata o processo de inspeção realizado através das OORTS, apresenta a análise dos resultados, apoiada pela estatística descritiva, e discute os resultados obtidos.

A seção 2 descreve o processo de inspeção através das OORTs, apresentando os principais trabalhos relacionados ao tema. A seção 3 apresenta a definição da pesquisa, incluindo aspectos de sua realização. A seção 4 descreve a análise de dados efetuada, destacando os resultados identificados. As conclusões são apresentadas na seção 5.

\section{Object-Oriented Reading Techniques (OORTs)}

\subsection{Trabalhos Relacionados}

As técnicas de inspeção [Fagan, 1986] vêm sendo empregadas, com sucesso, para identificar defeitos em diferentes tipos de artefatos de software, tais como documentos de requisitos, especificações preparadas através de diversos métodos (como modelo 


\section{Simpósio Brasileiro de Qualidade de Software}

entidade-relacionamento, análise estruturada e orientação a objetos), listagem do código do sistema em diferentes linguagens, etc. A inspeção tem se mostrado eficiente e eficaz, uma vez que auxilia a detecção de grande parte dos defeitos presentes no artefato, a um custo aceitável.

Em particular, as técnicas de inspeção baseadas em leitura [Basili, 1996] têm sido utilizadas em uma quantidade significativa de projetos. Conrad (2003) relata a existência de cerca de 150 estudos publicados sobre a utilização destas técnicas, sendo destacados os seguintes resultados: (a) a inspeção aplicada ao código do sistema reduz os custos do projeto em até $39 \%$ e a inspeção aplicada ao projeto reduz esses custos em até $44 \%$; (b) a produtividade no desenvolvimento de software tem um acréscimo de $30 \%$ a $50 \%$ e o tempo de desenvolvimento tem uma redução de $10 \%$ a 30\%; há experiências que indicam uma economia de $20 \%$ no esforço total de desenvolvimento, através da inspeção aplicada a documentos do projeto do sistema.

Atualmente, cada vez mais empresas vêm empregando a UML como linguagem de especificação e projeto de alto nível. Desta forma, é de grande importância o investimento em técnicas de inspeção adaptadas para leitura dos diagramas UML. As técnicas Object-Oriented Reading Techniques (OORT) propostas por pesquisadores da Universidade de Maryland [Travassos, 1999], compõem um processo de inspeção de especificações modeladas em UML, que já conta com alguns estudos e experiências [Bunde, 2002; Conrad, 2003].

No entanto, poucos são os estudos, divulgados na literatura, com a participação de analistas de sistemas como inspetores, uma vez que grande parte dos trabalhos sobre inspeção utiliza estudantes como sujeitos das pesquisas.

Uma experiência de emprego das técnicas OORT no ambiente empresarial foi realizada na Erickson, Noruega [Bunde, 2002], utilizando uma versão das OORTs, com algumas adaptações consideradas necessárias para atender às peculiaridades do ambiente industrial. Os resultados obtidos foram analisados e apresentados, destacandose as seguintes conclusões: (a) as técnicas OORT mostraram-se eficientes e auxiliaram a detectar mais do que duas vezes mais defeitos do que a técnica de inspeção anteriormente utilizada na empresa; (b) o experimento apontou para algumas modificações nas OORTs que, se realizadas, poderiam melhorar os resultados obtidos.

Os trabalhos já realizados, destacados acima, sugerem a necessidade de se conduzir novos estudos empíricos, a fim de se avaliar a viabilidade prática de utilização das OORTs por analistas de sistemas que atuam no ambiente empresarial. Além disso, nota-se a necessidade de se obter subsídios para realização de melhorias, complementações e adaptações nas OORTs, buscando obter resultados mais eficientes e eficazes das inspeções dos artefatos em UML.

\subsection{OORTs}

As OORTs são resultado dos trabalhos de uma equipe de pesquisadores da Universidade de Maryland, que adaptaram as tradicionais técnicas de leitura, definindo um processo para inspeção de documentos UML [Travassos, 1999]. Posteriormente, surgiram extensões, entre as quais se destacam as realizadas por Bunde e Pedersen em um estudo realizado na empresa Ericsson [Bunde, 2002; Conrad, 2003]. Os trabalhos destes 


\section{Simpósio Brasileiro de Qualidade de Software}

autores forneceram a base principal para a definição das OORTs adotadas no presente trabalho.

O processo de inspeção através das OORTs compreende sete tipos de leitura, classificadas em leitura horizontal e leitura vertical [Travassos, 1999], que são realizadas com a finalidade de detectar defeitos nos modelos UML. As leituras verticais visam comparar artefatos resultantes da mesma fase de desenvolvimento do software, como, por exemplo, comparação entre diagrama de classes e diagrama de estados, elaborados na fase de projeto lógico. As leituras verticais propõem-se a comparar artefatos resultantes de diferentes fases do desenvolvimento do software, tais como requisitos e projeto. Enquanto as técnicas horizontais prestam-se principalmente à análise de consistência entre os diagramas, as técnicas verticais favorecem a análise de completeza e rastreabilidade dos diagramas comparados. Os dois tipos de leitura são ilustrados na Figura 1 e sumarizados a seguir.

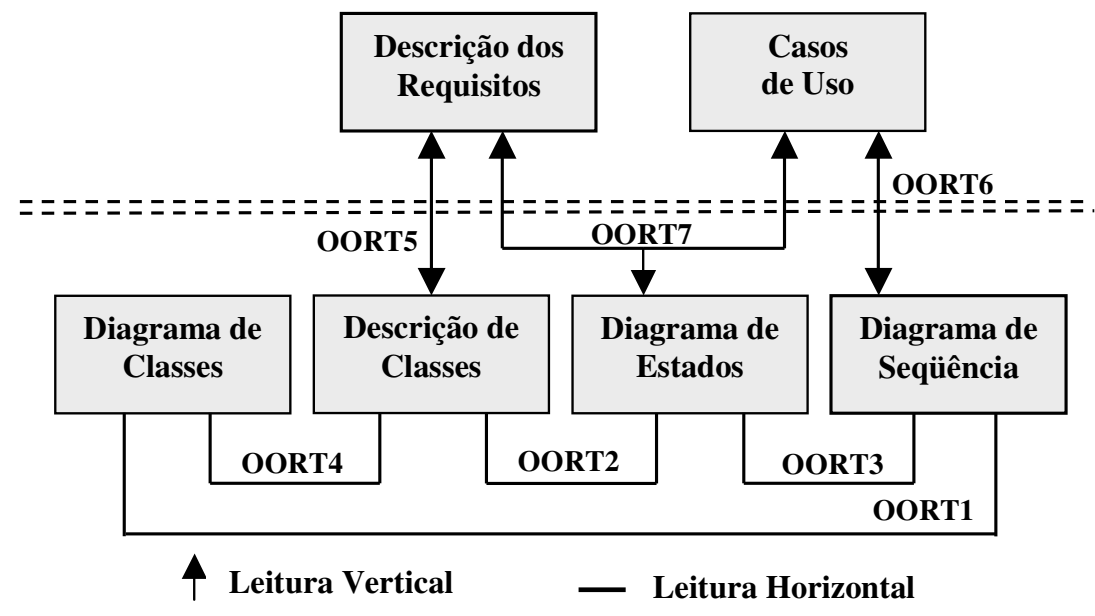

Figura 1. As OORTs e os Diagramas e Artefatos a elas Relacionados

- Leitura Horizontal. Compreende as quatro leituras destacadas a seguir.

- OORT1. Analisa um Diagrama de Sequiência comparativamente a um Diagrama de Classes, com o objetivo de verificar se o Diagrama de Classes do sistema descreve as classes e seus relacionamentos, de tal forma que o comportamento especificado no Diagrama de Seqüência esteja corretamente capturado.

- OORT2. Analisa um Diagrama de Estados em relação a uma Descrição de Classes, com o objetivo de verificar se as classes estão definidas, para que elas possam capturar a funcionalidade especificada pelo Diagrama de Estados.

- OORT3 - Analisa um Diagrama de Seqüência comparado a um Diagrama de Estados, com o objetivo de verificar se toda transição de estado para um objeto pode ser atingida pelas mensagens transmitidas e recebidas por aquele objeto.

- OORT4. Analisa um Diagrama de Classes em relação a uma Descrição de Classes, com o objetivo de verificar se a descrição detalhada das classes possui toda a informação necessária e se a descrição das classes está correta. 


\section{Simpósio Brasileiro de Qualidade de Software}

- Leitura Vertical. Compreende as três leituras destacadas a seguir.

- OORT5. Analisa uma Descrição de Classes comparativamnte à Descrição de Requisitos, com o objetivo de verificar se os conceitos e serviços que são descritos pelos requisitos funcionais são capturados pela descrição da classe.

- OORT6. Analisa um Diagrama de Sequiência em relação a um Diagrama de Casos de Uso, buscando verificar se os Diagramas de Seqüência descrevem uma combinação apropriada de objetos e mensagens que capturam a funcionalidade da especificação dos Casos de Uso.

- OORT7. Analisa um Diagrama de Estados comparado à Descrição de Requisitos e ao Diagrama de Casos de Uso, com o objetivo de verificar se os Diagramas de Estados descrevem estados apropriados dos objetos e eventos que disparam mudanças de estados, como descrito pela especificação dos Casos de Uso.

As técnicas cobrem quase todos os diagramas UML. Além disso, a Descrição dos Requisitos é usada para verificar se os diagramas atendem à especificação do sistema. Cada técnica compara pelo menos dois artefatos, visando detectar defeitos neles. É importante destacar que as técnicas pressupõem que tanto a Descrição de Requisitos quanto o Diagrama de Casos de Uso são corretos e livres de defeitos.

Cada técnica consiste de uma série de passos e de um checklist, que dirigem os inspetores na leitura dos diagramas e detecção de defeitos nos mesmos. Os defeitos detectados são classificados, quanto ao tipo, em:

- Omissão;

- Informação Estranha;

- Fato Incorreto;

- Ambigüidade;

- Inconsistência.

Quanto ao grau de criticalidade, cada defeito é classificado em:

- Sério, quando se refere a problema que inviabiliza a continuidade da leitura, indicando que o sistema deve ser re-especificado;

- Média Criticalidade, quando o defeito invalida a porção da especificação e os diagramas enfocados na técnica;

- Não sérios, quando não comprometem os diagramas tratados, apenas indicando, por exemplo, a necessidade de complementações.

As OORTs são apresentadas em detalhes em Cruz (2005). 


\section{Simpósio Brasileiro de Qualidade de Software}

\section{Definição e Realização da Pesquisa}

As perguntas que o estudo buscou responder foram: Qual é o nível de eficiência das técnicas OORT, em termos de quantidade de defeitos detectados em especificações de software representadas através de Unified Modeling Language? E qual é o nível de satisfação dos especialistas que inspecionaram a especificação UML através das técnicas OORT, em relação a aspectos importantes envolvidos na aplicação dessas técnicas?

O estudo propunha-se a envolver profissionais com experiência em especificação de sistemas de software através de UML. Dada a dificuldade de se obter profissionais que conhecem e possuem experiência em UML, o estudo baseou-se em uma amostra por conveniência, definida com base no critério "experiência mínima de 2 anos com UML". Foram identificados 7 analistas de sistemas que atendiam ao critério estipulado e atuavam na região de Piracicaba, que foram contactados e concordaram em participar da pesquisa.

Foi solicitado que os participantes inspecionassem um Sub-Sistema de Controle de Matrículas, que faz parte de um Sistema de Administração Acadêmica de Ensino Superior, especificado em UML. Para viabilizar a consecução dos objetivos do estudo, que envolvia a detecção de defeitos nos artefatos UML, foram injetados defeitos representativos de todos os tipos considerados, em todos os diagramas. Ao todo, foram injetados 47 defeitos, relacionados aos tipos constantes da classificação adotada omissão, fato incorreto, inconsistência, ambigüidade e informação estranha..

Cada participante recebeu os seguintes documentos para a inspeção:

- Especificação do Sub-Sistema de Matrícula Acadêmica, elaborada em UML, contendo: Descrição informal dos requisitos do sistema; Diagrama de Casos de Uso, acompanhado da respectiva descrição; Diagrama de Classes, acompanhado da Descrição das Classes; Diagrama de Sequiência; e Diagrama de Estados.

- Descrições da sete OORTs, cada qual apresentada através de um formulário, no qual são listados os passos para aplicação da técnica.

- Formulário de Anotação de Defeitos para Leitura Horizontal, que permitia aos participantes anotar, durante cada leitura horizontal: tipo do defeito detectado, palavra-chave relativa ao defeito, tipo de discrepância a que se refere o defeito, classificação do defeito, grau de severidade do defeito, identificação do requisito relacionado ao defeito, questão relativa ao defeito.

- Formulário de Anotação de Defeitos para Leitura Vertical, que permitia aos participantes anotar, durante cada leitura vertical: tipo do defeito detectado, palavrachave relativa ao defeito, tipo de discrepância a que se refere o defeito, classificação do defeito, grau de severidade do defeito, identificação do requisito relacionado ao defeito, questão relativa ao defeito.

- Tabela de Classificação de Defeitos possíveis de ocorrer nos artefatos do sistema, destacando os seguintes tipos de defeitos, devidamente definidos: omissão, fato incorreto, inconsistência, ambigüidade e informação estranha.

- Formulário de Relatos sobre o Uso das Técnicas OORT, compreendendo 50 questões que buscavam identificar aspectos relacionados à usabilidade (entendimento das 


\section{Simpósio Brasileiro de Qualidade de Software}

técnicas, facilidade de preenchimento dos formulários), completeza e consistência das OORTs.

Primeiramente, foi realizado um teste piloto com um analista de sistemas, em setembro de 2004, com o objetivo de verificar se os documentos preparados para a pesquisa eram adequados. Os resultados deste teste indicaram a necessidade de se promover algumas correções nos documentos utilizados na pesquisa, incluindo: melhorar as definições dos tipos de defeitos apresentadas na Tabela de Classificação de Defeitos; e deixar mais claro os checklists constantes das OORTs. Com base nestes resultados, os documentos utilizados foram revistos e melhorados.

A pesquisa foi realizada entre dezembro de 2004 e fevereiro de 2005. Inicialmente, cada participante foi contactado por telefone e, posteriormente, todos receberam os documentos da pesquisa através de e-mail. Após o envio dos documentos, foram agendadas reuniões individuais, no local de trabalho do participante, para o fornecimento de esclarecimentos necessários sobre o processo de realização da inspeção.

É importante destacar que, dos 7 participantes contactados, 2 não devolveram os formulários preenchidos, sendo, portanto eliminados da pesquisa. Os demais participantes realizaram a inspeção do sistema e responderam o Formulário de Relatos sobre o Uso das OORTs.

\section{Análise dos Resultados}

Os dados coletados através dos formulários foram tabulados e posteriormente tratados através da estatística descritiva (Pereira, 2004), de forma compatível com o número de participantes da pesquisa.

Dos 5 profissionais que realizaram a inspeção (identificados como "especialistas"), um deles possuía 3 anos de experiência em utilização de UML para especificação de software; os demais possuíam mais de 4 anos de experiência. Além disso, todos já tinham alguma experiência em inspeção de software, porém nenhum deles havia utilizado as técnicas OORT.

A seguir será apresentada, inicialmente, a análise referente à eficiência das técnicas OORT na detecção de defeitos e, em seguida, a análise referente à avaliação das técnicas por parte dos especialistas.

\subsection{Eficiência das Técnicas OORT}

Os dados obtidos mostraram que, dos 47 defeitos injetados nos artefatos, $34(72,4 \%)$ foram detectados através da inspeção feita pelos especialistas.

A Figura 2 apresenta os resultados obtidos, em termos de detecção dos defeitos através de cada OORT ${ }^{1}$.

\footnotetext{
${ }^{1}$ As Figuras 2, 3 e 4 apresentam, além dos gráficos de barras referentes à quantidade de defeitos detectados, as curvas baseadas nas porcentagens de defeitos detectados.
} 


\section{Simpósio Brasileiro de Qualidade de Software}

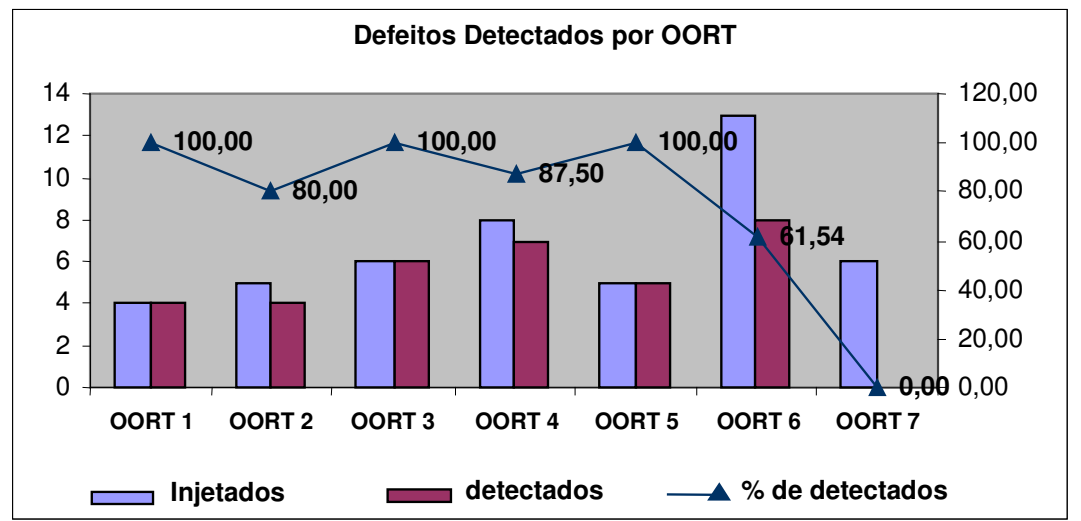

Figura 2. Defeitos Existentes e Detectados por OORT

Na Figura 2, pode-se observar que, em três casos - utilizando-se a OORT1, a OORT3 e a OORT5, ocorreu a detecção de $100 \%$ dos defeitos existentes nos artefatos UML inspecionados. Observa-se também que, dos 6 defeitos existentes que deveriam ser detectados através da OORT7, nenhum deles foi detectado. Cabe destacar, aqui, que o fato de um especialista não ter realizado a parte da inspeção relacionada com o uso da OORT7 contribuiu para este resultado.

A Figura 3 apresenta os resultados obtidos, em termos de detecção dos defeitos, considerando-se a classificação de defeitos adotada. Conforme pode ser observado nesta figura, os maiores percentuais de defeitos encontrados ocorreram para as classificações de Inconsistência (88,24\%), Ambigüidade (100\%) e Informação Estranha (75\%), ocorrendo também um índice significativo para defeitos de Omissão (50\%). O índice mais baixo ocorreu para a detecção de defeitos do tipo Fato Incorreto $(37,50 \%)$.

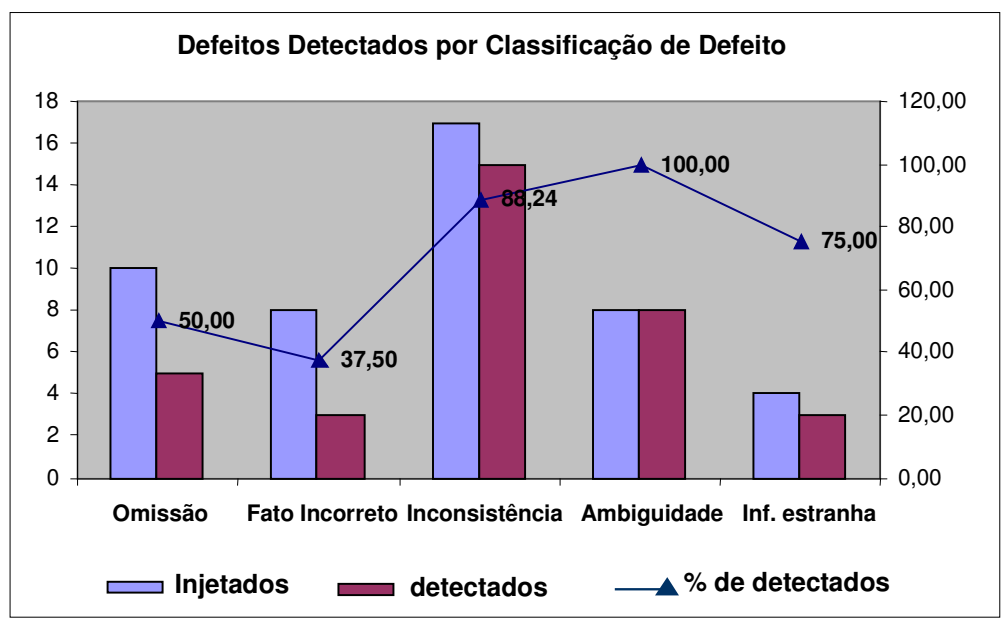

Figura 3. Defeitos Injetados e Detectados por Classificação 


\section{Simpósio Brasileiro de Qualidade de Software}

A Figura 4 mostra os dados obtidos, em termos de defeitos detectados por meio da Leitura Horizontal (OORT 1, OORT 2, OORT 3 e OORT 4) e da Leitura Vertical (OORT 5, OORT 6 e OORT 7). Pode-se observar, pela Figura, que um percentual elevado de defeitos foi detectado por meio da Leitura Horizontal, atingindo 91,30\%. O percentual de defeitos detectados através da Leitura Vertical foi mais baixo, atingindo $54,17 \%$.

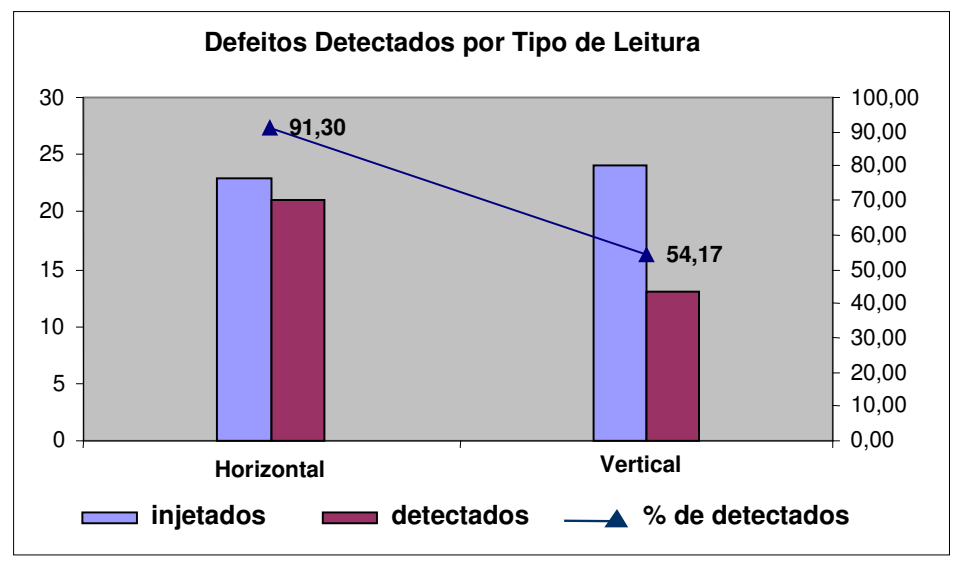

Figura 4. Defeitos Injetados e Detectados nas Leituras Horizontal e Vertical

Outros índices foram calculados, cruzando-se os dados referentes a tipos de defeitos, técnica OORT e tipo de leitura. Tais índices não serão apresentados neste artigo devido à restrição de espaço, mas podem ser obtidos com os autores.

\subsection{Avaliação das Técnicas OORT}

São apresentados, a seguir, os principais resultados obtidos a partir das respostas dadas pelos especialistas a questões do Formulário de Relatos sobre o Uso das Técnicas OORT.

A Figura 5 corresponde à avaliação da classificação de defeitos utilizada na inspeção e às descrições fornecidas para cada tipo de defeito, na Tabela de Classificação de Defeitos ${ }^{2}$.

2 Nas Figuras 5 a 8 , os gráficos de barra mostrados baseiam-se nas respostas fornecidas pelos especialistas às questões do Formulário de Relatos indicadas à esquerda do gráfico. 


\section{Simpósio Brasileiro de Qualidade de Software}

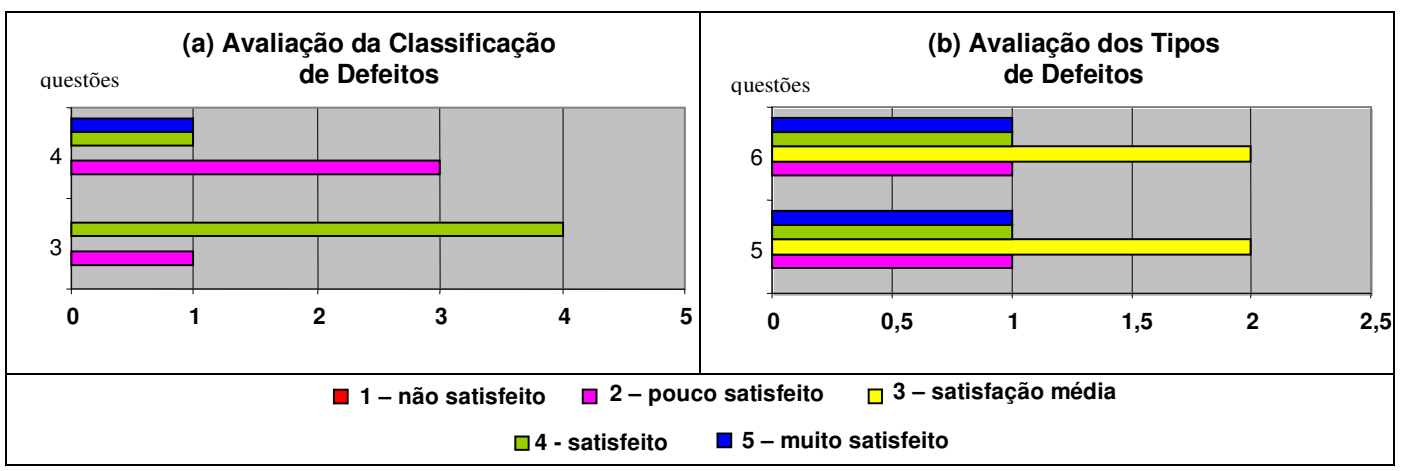

Figura 5. Avaliação da Classificação e da Descrição dos Tipos de Defeitos

A parte (a) da Figura 5 mostra a avaliação da Tabela de Classificação de Defeitos em termos do entendimento da classificação (questão 3) e de sua completude (questão 4). Quanto ao entendimento, 80\% dos especialistas declararam estar "satisfeitos" e os $20 \%$ restantes declararam estar "pouco satisfeitos". Quanto à completude, $60 \%$ dos especialistas informaram estar "pouco satisfeitos", 20\% "satisfeitos" e 20\% "muito satisfeitos". Não ocorreram respostas referentes aos níveis "não satisfeitos" e "satisfação média".

A parte (b) da Figura 5 mostra os resultados obtidos na avaliação da descrição dos Tipos de Defeitos, incluindo os aspectos de entendimento (questão 5) e de completude (questão 6). Verifica-se que a categoria de "média satisfação" foi indicada por $40 \%$ dos especialistas, sendo que dos demais, $40 \%$ estão divididos entre as categorias de "satisfeitos" e de "muito satisfeitos". Além disso, 20\% dos especialistas declararam estar "pouco satisfeitos" com a descrição dos tipos de defeitos utilizada na inspeção.

A Figura 6 apresenta quatro gráficos, cada qual referente à avaliação de cada uma das OORTs que compõe a Leitura Horizontal (OORT1, OORT2, OORT3 e OORT4). Para esta avaliação, foi solicitado que os especialistas indicassem o nível de satisfação com os checklists de cada OORT, considerando os aspectos de compreensão (questões 19, 23, 27 e 31), necessidade (questões 20, 24, 28 e 32) e suficiência (questões 21, 25, 29 e 33) das questões existentes nos checklists e, além disso, de suficiência dos artefatos abordados nos checklists para aplicar uma OORT (questões 22, 26, 30 e 34). 


\section{Simpósio Brasileiro de Qualidade de Software}

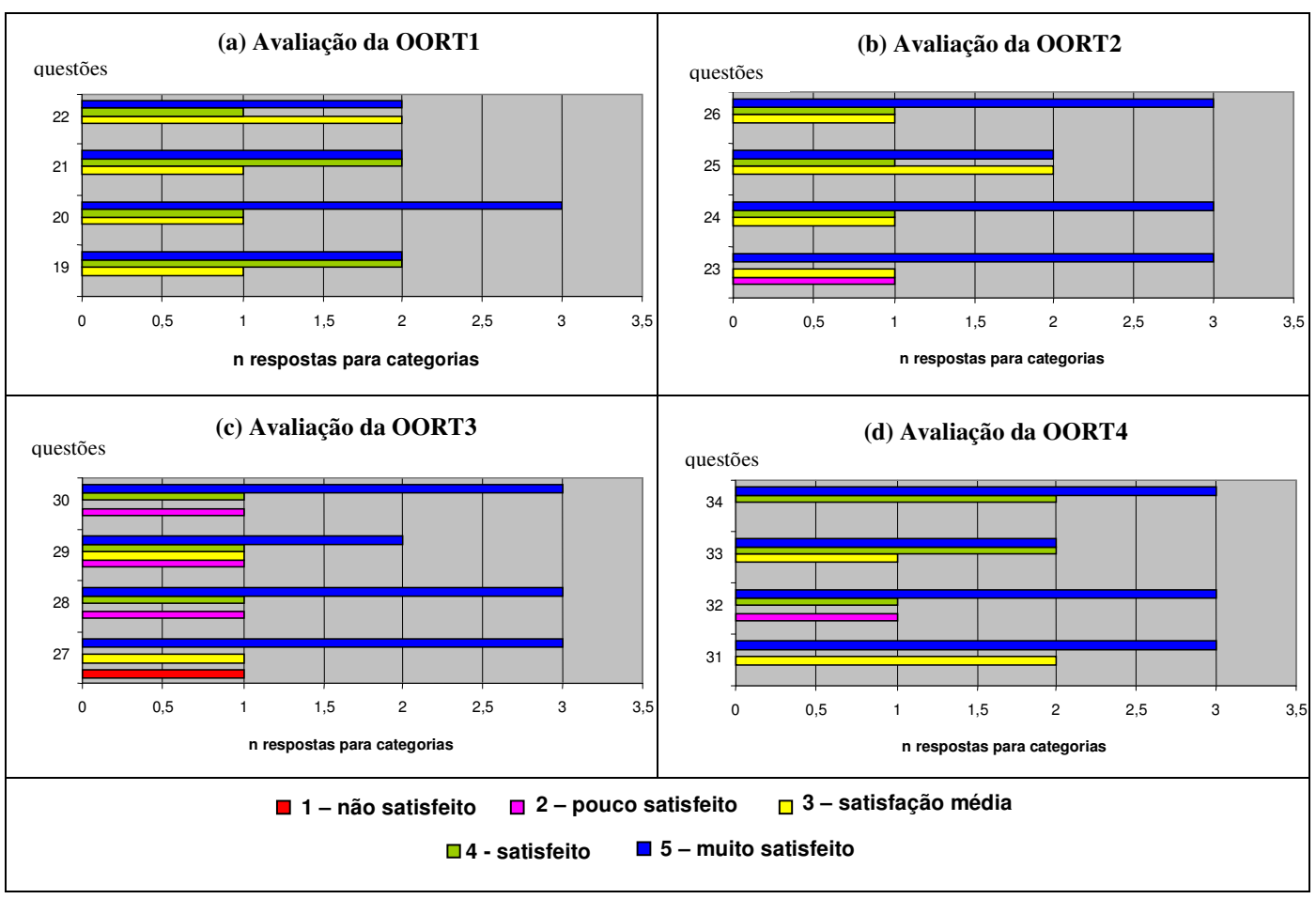

Figura 6. Avaliação das OORTs da Leitura Horizontal

Observando-se os resultados na Figura 6, para todas as questões analisadas, é perceptível que as categorias de "satisfação" e "muita satisfação" são predominantes, ocorrendo para todas um índice de no mínimo $40 \%$ de "muita satisfação". No entanto, é importante salientar que índices de "pouca satisfação" e de "não satisfação", atingindo o valor de $20 \%$, apareceram em relação às OORTs 2,3 e 4, destacando-se a OORT 3, para a qual pelo menos um destes dois índices foi apontado, conseqüentemente sendo a OORT3 a que recebeu menor nível de satisfação, na Leitura Horizontal.

A Figura 7 apresenta três gráficos, cada qual referente a uma OORT das que compõem a Leitura Vertical (OORT5, OORT6 e OORT7). Para esta avaliação, foi solicitado que os especialistas indicassem o nível de satisfação com cada OORT, considerando os aspectos de compreensão (questões 35, 39 e 43), necessidade (questões 36, 40 e 44) e suficiência (questões 37, 41 e 45) das questões apresentadas e, além disso, de suficiência dos artefatos fornecidos para aplicar uma OORT (questões 38, 42 e 46).

Conforme mostra a Figura 7, a OORT5 recebeu pelo menos $40 \%$ de indicações de "muita satisfação" por parte dos especialistas, para todos os aspectos avaliados, não tendo recebido avaliações inferiores a "média satisfação". A OORT6 e a OORT7 não obtiveram uma avaliação tão boa quanto a da OORT 5, pois apesar de terem apresentado, na maioria das perguntas, um índice referente a "muito satisfeito" bem presente, ainda assim não se pode ignorar o fato de que, em todas as perguntas, ocorreram as categorias de "pouca satisfação" ou de "não satisfação", com um mínimo de $20 \%$. 


\section{Simpósio Brasileiro de Qualidade de Software}

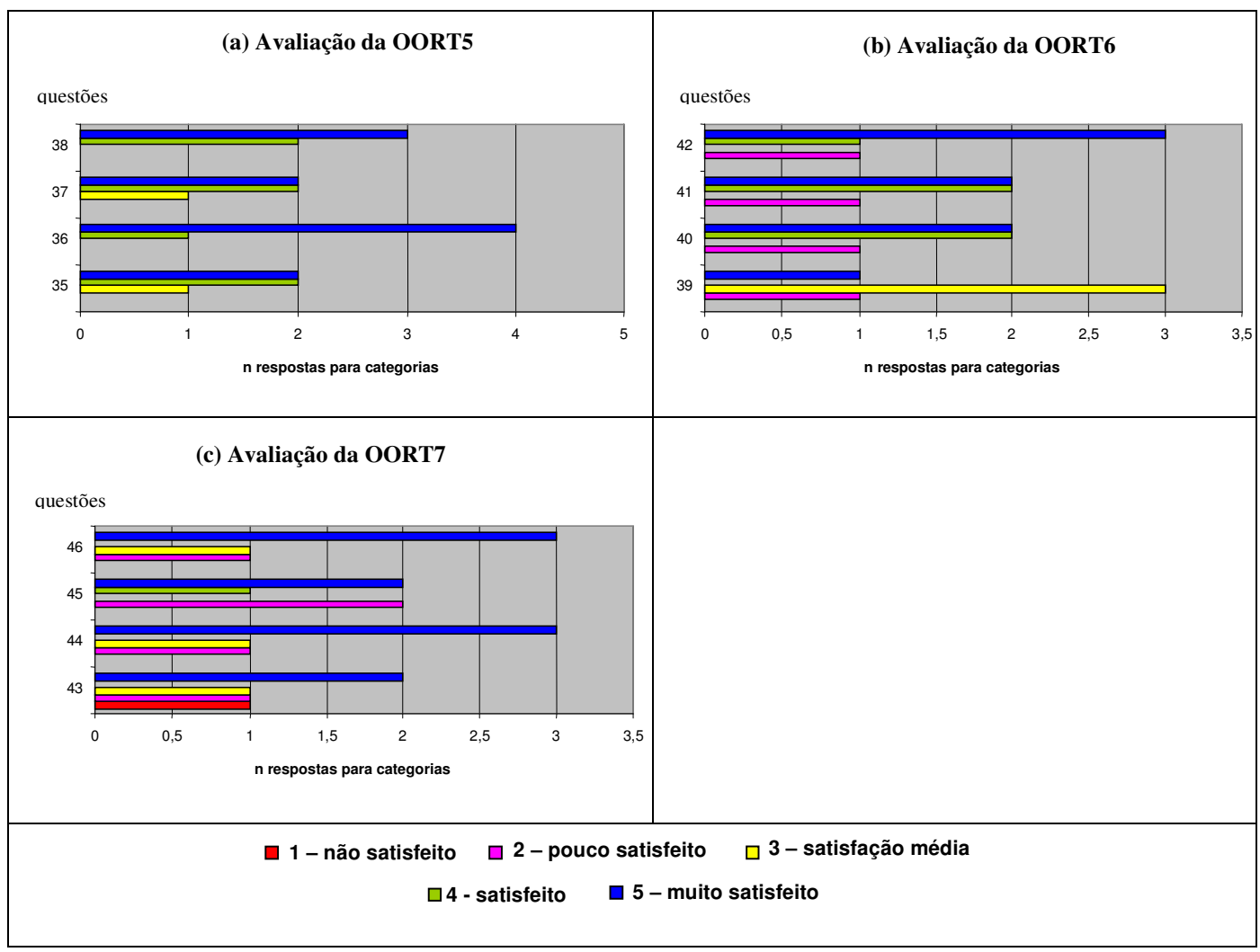

Figura 7. Avaliação das OORTs da Leitura Vertical

A Figura 8 apresenta os resultados referentes ao aumento de conhecimento propiciado pela inspeção aos especialistas e à opinião expressada pelos especialistas a respeito deles utilizarem as técnicas OORT em futuros projetos de software.

O acréscimo de conhecimento, trazido pela realização da inspeção utilizando as técnicas OORT, foi avaliado através de duas questões, que abordavam conhecimento sobre inspeção (questão 48) e sobre UML (questão 49). Os resultados apresentados na Figura 8 a sugerem que $100 \%$ dos especialistas tiveram seu conhecimento sobre inspeção aumentado (40\% de "satisfação média", 40\% "satisfeitos" e 20\% "muito satisfeitos") Quanto ao aumento do conhecimento sobre UML, 40\% dos especialistas manifestaram-se "pouco satisfeitos", $40 \%$ com "satisfação média" e $20 \%$ "satisfeitos". Ou seja, os especialistas beneficiaram-se mais com os conhecimentos adquiridos sobre inspeção (incluindo conhecimento sobre as técnicas OORT) do que com os conhecimentos sobre UML (categoria na qual eram especialistas).

Como última questão, foi indagado aos especialistas se estes consideravam, de alguma forma, a possibilidade de utilizar as técnicas OORT em um projeto de software. A Figura 8b apresenta os resultados obtidos a partir desta pergunta, que indicam que $60 \%$ dos especialistas posicionaram-se favoravelmente à utilização das técnicas OORT (incluindo as respostas referentes a "muito satisfeitos", "satisfeitos" e "satisfação média"). Verificou-se também que $40 \%$ dos especialistas manifestaram-se "pouco 


\section{Simpósio Brasileiro de Qualidade de Software}

satisfeitos" com a possibilidade de utilizar as OORTs em projetos futuros. É importante destacar que dois participantes declararam textualmente que seria difícil aplicar as técnicas OORT em projetos reais, no ambiente empresarial, devido ao nível de detalhe envolvido nas técnicas e à extensão e quantidade de diagramas UML de um projeto real.

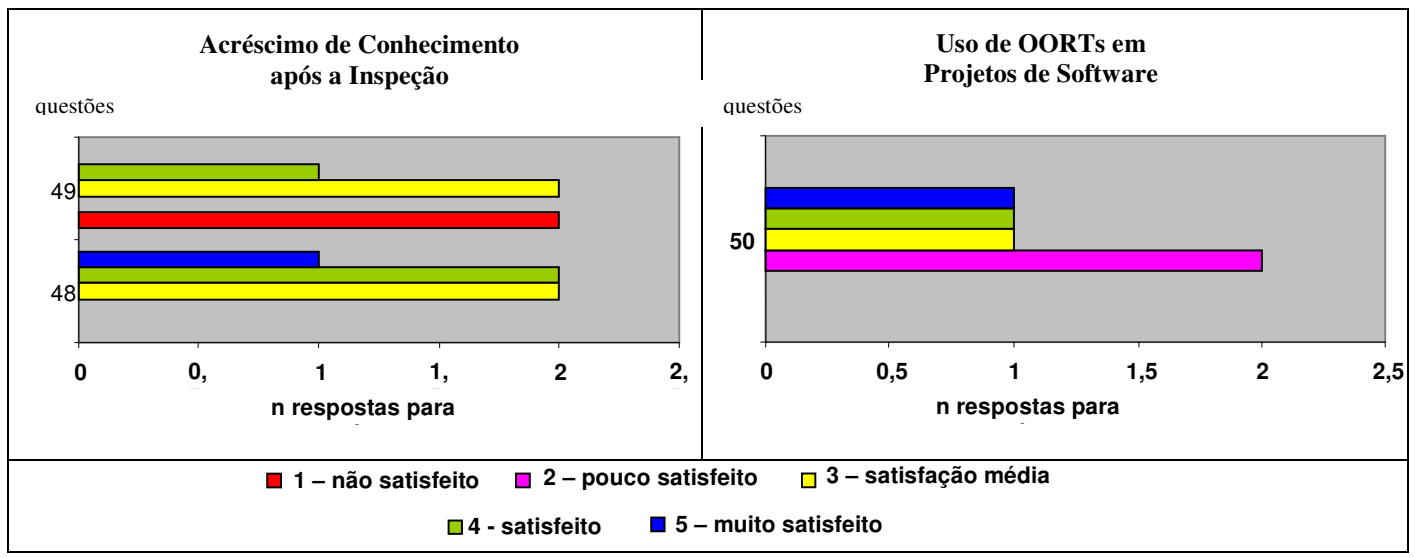

Figura 8. Aumento de Conhecimento e Uso de OORTs em Projetos Futuros

\section{Considerações Finais}

Este trabalho apresentou um estudo empírico sobre a avaliação das técnicas OORT, conduzido com o objetivo de avaliar tanto a eficiência das técnicas, em termos de detecção de defeitos, quanto fatores relacionados à utilização dessas técnicas por analistas de sistemas de instituições da região de Piracicaba, estado de São Paulo.

A partir da análise dos resultados, ficaram evidenciados os seguintes pontos:

- Quanto à eficiência das técnicas OORT, pode-se concluir que, para a pesquisa realizada, incluindo os especialistas que dela participaram e o sistema inspecionado:

- De maneira geral, as técnicas OORT mostraram-se significativamente eficientes, uma vez que $72,4 \%$ dos defeitos contidos na especificação do sistema, preparada através dos diagramas UML, foram detectados.

- Considerando-se as sete técnicas, a técnica OORT7 mostrou-se menos eficiente, uma vez que os defeitos que deveriam ser encontrados através dela não foram detectados pelos participantes.

- Considerando-se os cinco tipos de defeitos adotados, o índice de detecção de defeitos mais baixo ocorreu para os defeitos referentes a "informação estranha".

- Considerando-se os dois tipos de leitura, a Leitura Horizontal mostrou-se significativamente mais eficiente do que a Leitura Horizontal, na detecção dos defeitos relacionados a cada uma delas, sendo que a Leitura Horizontal detectou 91,30\% dos defeitos e a Leitura Vertical detectou 54,17\% dos defeitos.

- Quanto à avaliação das características dos documentos e formulários que compuseram as técnicas OORT, pode-se concluir que, para a pesquisa realizada, incluindo os especialistas que dela participaram e o sistema inspecionado: 


\section{Simpósio Brasileiro de Qualidade de Software}

- Considerando-se a classificação de defeitos utilizada, a grande maioria (80\%) dos participantes declarou estar satisfeita quanto ao entendimento propiciado pela classificação. No entanto, apenas $20 \%$ informou estar satisfeitos com a completude da classificação adotada. Tais resultados podem sugerir a necessidade de se revisar e testar mais detalhadamente a classificação, buscando estendê-la de forma a possibilitar a cobertura de outros tipos de defeitos que porventura possam ocorrer.

- Considerando-se a descrição fornecida a cada tipo de defeito constante na classificação adotada, $80 \%$ dos participantes declararam estar "muito satisfeitos", "satisfeitos" ou com "satisfação média", tanto em relação ao quesito de entendimento quanto ao quesito de completude. Tal resultado pode ser um indicativo de que, de maneira geral, os defeitos estão definidos adequadamente.

- Considerando-se as OORTs que compõem a Leitura Horizontal, constatou-se que a OORT 3 (que analisa comparativamente Diagrama de Sequiência x Diagrama de Estados) foi a que recebeu um menor nível de satisfação, conforme declarado pelos especialistas (20\%, somando "pouca satisfação" e "não satisfação"). Tal resultado pode indicar a necessidade de se revisar a OORT3, objetivando melhorar sua qualidade.

- Considerando-se as OORTs que compõem a Leitura Vertical, identificou-se que a OORT6 e a OORT7 receberam um índice menor referente á satisfação dos participantes, em relação à OORT5. De maneira geral, a OORT7 foi indicada como a de menor índice de satisfação por parte dos especialistas. Tais resultados podem ser vistos como um indicativo para se revisar as duas OORTs, especialmente a OORT7, a fim de se promover melhorias nas mesmas.

- Considerando-se o conhecimento propiciado aos especialistas através da participação na pesquisa, $100 \%$ dos participantes declararam que seu conhecimento sobre inspeção (incluindo o uso das técnicas OORT) foi incrementado. Tal resultado é bastante significativo e pode sugerir que a abordagem seguida na pesquisa seria útil para a definição de treinamento de profissionais para uso das técnicas OORT.

- Considerando-se a propensão demonstrada pelos especialistas em utilizar as técnicas OORT em um projeto de software, no ambiente empresarial, $40 \%$ dos participantes posicionaram-se desfavoravelmente a esta possibilidade. Tal resultado é significativo e aponta para a necessidade de se estabelecer estratégias de utilização das referidas técnicas, de maneira a tornar o processo de inspeção através das OORTs menos desgastante. É importante esclarecer que, na pesquisa, cada participante inspecionou toda a especificação do sistema, utilizando as sete OORTs. Uma estratégia diferente, em que cada profissional ficasse responsável pela aplicação de apenas uma parte das OORTs, tenderia a ser menos cansativa para os inspetores.

É importante salientar que o estudo empírico apresentado representou um passo inicial para um domínio mais aprofundado das técnicas OORT, não apenas visando identificar a eficiência dessas técnicas na detecção de defeitos em especificações elaboradas através de UML, mas também buscando identificar aspectos que poderiam ser melhorados nas técnicas, como foi abordado na presente pesquisa. 


\section{Simpósio Brasileiro de Qualidade de Software}

Como trabalho futuro, destaca-se a importância de se realizar uma nova pesquisa, envolvendo maior número de especialistas, o que tornaria possível a análise dos resultados através de técnicas de inferência estatística, levando, assim, a resultados passíveis de serem estendidos a um escopo maior referente à utilização das técnicas OORT.

Finalmente, o presente trabalho presta uma contribuição à melhoria da qualidade de software, através da discussão de técnicas de avaliação da qualidade de especificações de requisitos e da apresentação de um estudo empírico com resultados significativos.

\section{Agradecimentos}

Os autores agradecem os profissionais que participaram da pesquisa, realizando a inspeção e respondendo o questionário.

\section{Referências}

Basili, V.R., Caldiera, G., Lanubile, F., and Shull, F. (1996) "Studies on Reading Techniques", Proceedings of the 21th Annual Software Engineering Workshop, NASA-SEL-96-002, Greenbelt, MD, p. 59-65

Booch, G., Rumbaugh, J., Jacobson, I. (1999) "The Unified Modeling Language User Guide", Addison-Wesley.

Bunde, G.A., Pedersen, A. (2002) "Defect Reduction by Improving Inspection of UML Diagrams in the GPRS Project", MSc Thesis, Agder University College, Norway.

Conrad, R. et all. (2003) "Object-Oriented Techniques for Inspection of UML Models An Industrial Experiment", Proceedings of the European Conference on ObjectOriented Programming, Darmstadt, Germany, Springer-Verlag 2743, p. 483-501.

Cruz, Erik R. (2005) "Inspeção de Especificações de Requisitos de Software Modelados em Unified Modeling Language", Universidade Metodista de Piracicaba.

Fagan, M.E. (1976) "Design and Code Inspection to Reduce Errors in Program Development”, IBM Systems Journal, Vol. 15, Number 3, p. 182-211.

Fagan, M.E. (1986) "Advances in Software Inspections", IEEE Transactions on Software Engineering, Vol. 12, Number 7, p. 744-751.

Gilb, T., Graham, D. (1993) “Software Inspection”, Addison-Wesley.

Pereira, J.C.R. (2004) “Análise de Dados Qualitativos”, Editora da Universidade de São Paulo - EDUSP.

Porter, A., H., Votta, L. (1996) “A Review of Software Inspections", Advances in Computers, vol. 42, p. 40-76.

Travassos, G.H., Shull, F., Fredericks, M., Basili, V.R. (1999) "Detecting Defects in Object-Oriented Designs: Using Reading Techniques to Increase Software Quality", Proceedings of the OOPSLA'99, Denver, CO, (in ACM SIGPLAN Notices,34(10), Oct. 1999), p. 47-56. 